\title{
Diagnosis and monitoring of Alzheimer Disease with saliva biomarker BACE1 and Preliminary study on prevention of AD with mesenchymal stem cells and their secretome
}

\author{
SJ Kellner* \\ Mesencell Biotech GmbH and Mesencell Biotech Ltd, Zueberwangen, Switzerland \\ ${ }^{*}$ Correspondence to: Steven James Kellner, Mesencell Biotech GmbH and Mesencell Biotech Ltd, Ruetistrasse 4, 9523 Zueberwangen, Switzerland; Tel: +33 633 8132 79; \\ E-mail: science@mesencell-biotech.com
}

Received: March 07, 2018; Accepted: March 20, 2018; Published: March 25, 2018;

\begin{abstract}
Alzheimer's disease (AD) is characterized by the slow decline of cognition and functional abilities over time. The diagnosis for probable and possible AD relies principally on clinical criteria. The confirmation of the disease is made post-mortem by identifying extracellular senile plaques and intraneuronal fibrillary tangles in the brains of subjects with clinically defined dementia. However, the field critically lacks validated AD specific peripheral biomarkers to support the diagnosis in living patients or for early detection of patients at risk before symptoms appear. BACE1 (Beta site amyloid precursor protein cleaving enzyme 1) cleaves Amyloid Precursor Protein (APP) at two beta sites and represents a key target enzyme in the monitoring and possible treatment of AD. The secretome of adipose derived mesenchymal stem cells contain amyloid beta degrading Neprilysin and ubiquitin carboxyl-terminal hydrolase L1 (UCH-L1), both known factors in influencing Alzheimer Disease.

In our preliminary study, we evaluated the usefulness of salivary BACE1 to determine risk to develop AD in clinically normal patients and the influence of the secretome of MSCs on BACE1 levels in saliva. A preliminary in house laboratory grading system for BACE1 content in saliva was established with high levels of salivary BACE1 present in older patients and putatively at risk to develop AD. Salivary BACE1 levels were significantly reduced in patients receiving intramuscular adipose tissue derived mesenchymal stem cell secretome.
\end{abstract}

BACE1 seems to be a useful biomarker to help diagnose $\mathrm{AD}$ and to monitor disease progression, and remarkable regression, when treated with stem cell secretome.

Keywords: Biomarkers, Alzheimer Disease, Mesenchymal stem cells, amyloid beta, amyloid precursor protein, amyloid plaques, BACE1, neprilysin, micro RNA, UHC-L1, disease monitoring, saliva

\section{Introduction}

Alzheimer's disease (AD) is characterized by the slow decline of cognition and functional abilities over time. The diagnosis for probable and possible $\mathrm{AD}$ relies principally on clinical criteria and neuropsychological tests defined by the National Institute of Neurological Disorders and Stroke (NINDS) and the Alzheimer's disease and Related Disorders Association (ADRDA) [1], mostly by assessment through a Mini-Mental State Examination MMSE. The confirmation of the disease is made post-mortem by identifying extracellular senile plaques and intraneuronal fibrillary tangles in the brains of subjects with clinically defined dementia. However, the field critically lacks validated AD specific peripheral biomarkers to support the diagnosis in living patients or for early detection of patients at risk before symptoms appear. Some investigators studied structural Magnetic Resonance Imaging [2] as means for in vivo confirmation, whereas others concentrate on liquid biomarkers, such as plasma amyloid beta (A $\beta)-40$ and 42 , albeit with mixed results [3].

$\mathrm{AD}$ is the most common form of dementia and is pathologically characterized by amyloid plaques, neurofibrillary tangles and loss of synapses in the brain. Neurofibrillary tangles consist of hyperphosphorylated tau protein while fibrils of the amyloid betapeptide (Ab) aggregate into amyloid plaques [4]. Smaller, oligomeric species of $\mathrm{Ab}$ have proven to be neurotoxic and cause synaptic dysfunction [5]. Ab is a proteolytic product of the amyloid precursor protein (APP) which is sequentially cleaved in an amyloidogenic pathway by beta- and gamma-secretases [4]. Beta- secretasecleaves APP close to the membrane, releasing a soluble APP beta-fragment (sAPPb). The remainingC-terminalfragment(APP-CTFb)canthenbecleavedbygammasecretase at different sites yielding Ab-peptides of varying length, Ab40 beingthemostcommon, whileAb42ismoretoxicand prone to aggregate. If APP is cleaved in a non-amyloidogenic pathway by alpha-secretase instead of beta-secretase, anon-toxicP3fragmentwillbeformedafterthe gamma-secretasecleavage.

Beta-site APP-cleaving enzyme 1 (BACE1), the beta-secretase in vivo, has been identified as a 501- amino acid residue single transmembrane aspartyl protease [6]. BACE1 cleaves APP at two beta sites and produces the C-terminal fragments (CTF) C99 and C89 [7]. C99 is subsequently cleaved by presenilin (PS)-dependent gamma-secretase complex to 
release Ab fragments. BACE1 therefore represents a key target enzyme in the monitoring and possible treatment of $\mathrm{AD}$. However, over the last decade, it has become clear that BACE1 proteolytically cleaves a number of substrates in addition to APP. When experimenting with total knock out BACE1 mice, the extreme reduction or total absence of BACE1 led to neurological dysfunctions [8]. Protocols using mesenchymal stem cells and/or their cell-free secretions (secretome) did not show these disadvantages, as, although many active ingredients are present and have shown to be beneficial, the concentration of these ingredients, the diverse mode of actions (i.e. anti-inflammatory molecules, content of alpha secretase neprilysin (NEP), antioxidants and ubiquitin carboxylterminal hydrolase L1) and long term but temporary cellular activation via microRNA prevent drastic changes. That being said, the content of amyloid beta degrading NEP and ubiquitin carboxyl-terminal hydrolase L1 (UCH-L1) in the secretome of mesenchymal stem cells (MSCs), have been investigated in some detail in preclinical in vitro and in vivo studies [9]. Also, on the production side of the secretome, both ingredients can be augmented through GMP compatible manufacturing processes. $\mathrm{UCH}-\mathrm{L} 1$ accelerates BACE1 degradation and affects APP processing and $\mathrm{Ab}$ production [10].

In our preliminary study, we evaluated a) the usefulness of salivary BACE1 to determine risk to develop AD in clinically normal patients andb) theinfluence of the secretome ofMSCs on BACE1 levels in saliva.

\section{Methods and Material}

\section{MSCs and secretome}

Mesenchymal stem cells were isolated from subcutaneous adipose tissue which had been harvested by mini liposuction under local anaesthesia from two healthy patients, undergoing this process for unrelated reasons. After digestion with collagenase, stem cells were isolated in culture flasks using a proprietary animal free stem cell attachment media. Cultivation of stem cells was done using a proprietary animal free stem cell growth media with minimal expansion after 1 passage to 3 million cells. Cells were identified by microscopy and with help of a CD marker panel (Abcam). Secretome was produced from these 3 million cells and analyzed for content in cytokines, neprilysin, micro and messenger RNA.

\section{Salivary samples}

Salivary samples of 8 Patients were taken first thing in the morning, at lunchtime and evening before eating and drinking. Sample size was approximately $1 \mathrm{ml} .200 \mathrm{ul}$ were mixed with same amount of protease inhibitor (Protease Inhibitor Cocktail, Sigma Aldrich, Switzerland). Samples with and without protease inhibition were either frozen immediately or left for 48 and 72 hours at room temperature before freezing them as well at minus 20 degrees Celsius before analysing.

Salivary samples of the 2 Patients receiving intramuscular injection of secretome 4 times every two to four weeks were taken one week after last injection in the morning before breaking fast and frozen without addition of protease inhibitor.

Saliva samples were not centrifuged or otherwise processed.

\section{ELISA}

Human Beta site APP cleaving enzyme 1 sandwich ELISA kit (Sun Long Biotech, China) was used. 10 ul salivary samples and diluted salivary samples (1: 1 with protease inhibitor) were processed according to standard procedure, measured at 450 OD and BACE 1 concentration in $\mathrm{pg} / \mathrm{ml}$ calculated with the help of the standard curve.

\section{Patients}

Patients were all healthy with no clinical signs of Alzheimer disease. Young patients were not showing any signs of mental deficiencies but some of the older Patients were self-assessed as having problems with concentration and memory. There were no family incidences of Alzheimer Disease.

\section{MMSE}

A German version of the mini-mental state examination was performed.

\section{Results}

\section{Mini-Mental State Examination}

MMSE was normal in all patients. However, two patients had selfassessed problems with concentration and memory, which the MMSE did not pick up.

\section{Saliva samples}

Saliva samples from morning, midday and evenings were analyzed (Table 1). In all samples BACE1 could be detected. There was no clear diurnal variation, but clear differences in samples in 7 out of 8 Patients regarding the time of sampling. Levels of BACE 1 varied from $267 \mathrm{pg} /$ $\mathrm{ml}$ to $939 \mathrm{pg} / \mathrm{ml}$.

Table 1. Diurnal variations to BACE 1 levels in saliva.

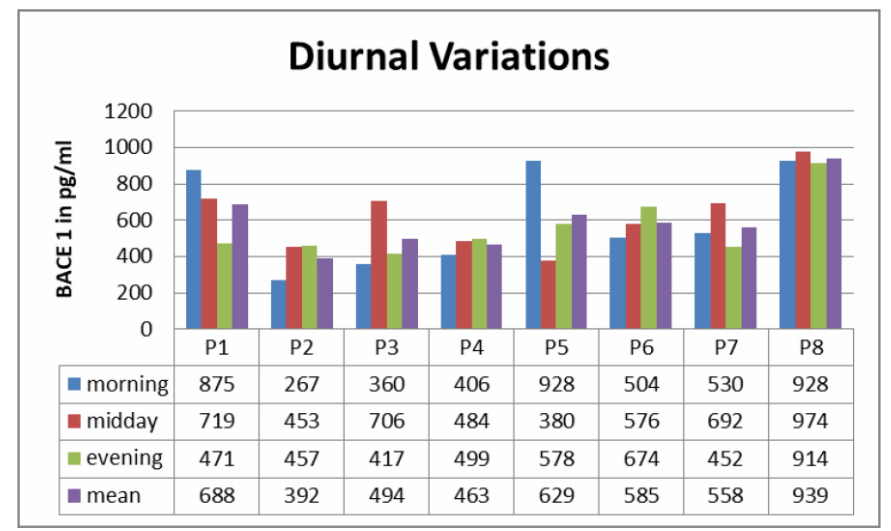

Saliva samples of three Patients were studies to see if BACE1 content at room temperature would change over 72 hours (Table 2). Fresh samples had a lower content of BACE1 then when measured after 48 respectively 72 hours.

Fresh saliva samples were compared with samples stabilized with protease inhibitors (Table 3). Results showed a big difference between the two groups. 
Table 2. BACE1 values in saliva according to time spent before analysis.

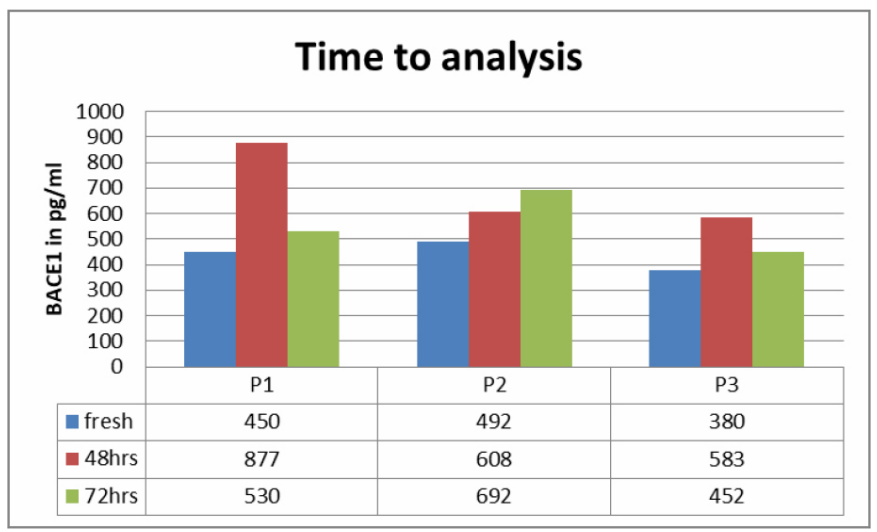

Table 3. Influence of protease inhibitor on BACE1 content in saliva samples.

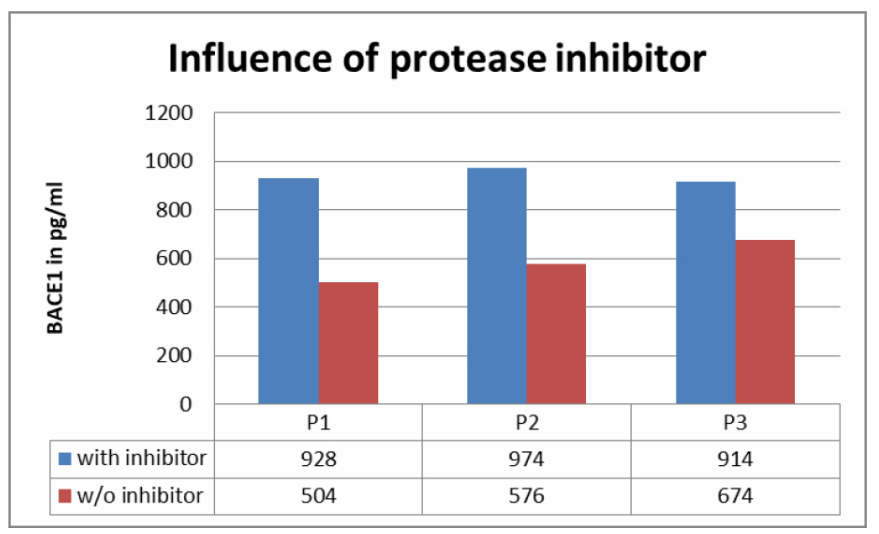

\section{ELISA}

ELISA tests were straight forward; however the small sample size of $10 \mathrm{ul}$ and the viscosity of saliva made it difficult to accurately and consistently dose the wells.

\section{Patients}

Our 8 patients were aged between 18 years of age and 62 years of age. The upper limit was meant to prevent including non-diagnosed early stages AD patients. There were 3 male and 5 female Patients. 2 patients, both female, were suspected to have a certain degree of memory problems without any influence on their MMSE.

\section{Stem cell treatment}

2 Patients had, unrelated to this study, treatments with the secretome of adipose derived MSCs. One female Patient was classified as at risk for $\mathrm{AD}$ with a higher than usual level of salivary BACE1 and beginning memory and concentration problems, the male Patient was within limits. Both showed a reduction in salivary BACE1 after treatment (Table 4). Sampling at different time during the day revealed big variations but were not consistent. It was felt that salivary samples before breakfast would reveal the most accurate values and also the highest values.
Table 4. Differences in salivary BACE1 after injections with secretome from MSCs.

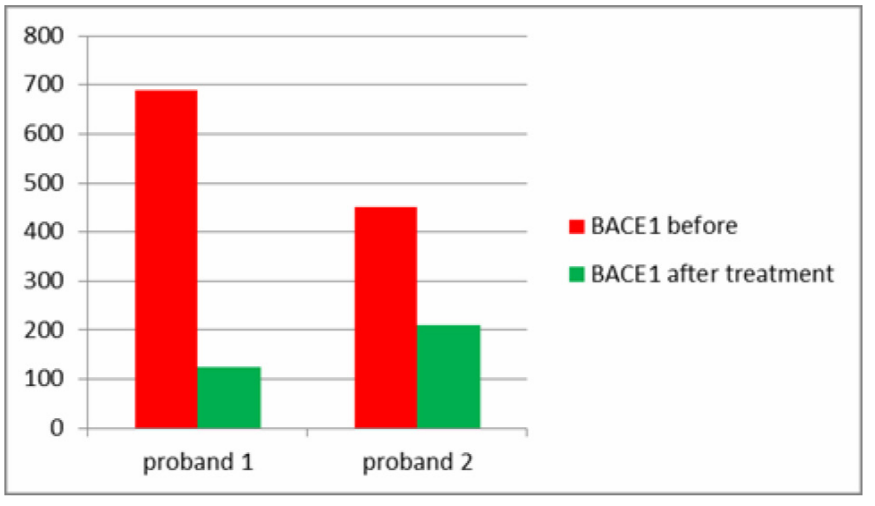

\section{Discussion}

Saliva samples are easily obtained, but variation in the amount of saliva is well known, with $\mathrm{AD}$ patients having more saliva production then normal controls. Theoretically, the total amount of saliva production and a weight factor would be needed for accuracy. Practically, although asked to be diligent, patients had quite a few variations in the time of sampling, making this in $\mathrm{AD}$ patients a difficult task. Time of delay between sampling and processing the samples revealed remarkable differences. The initial idea was that BACE1 would decline with passing hours before processing, which was not proven to be true. All the tested samples showed an increase of BACE1 values in the ELISA during the delay, however not linearly. This could be due to BACE1 not being freely suspended in saliva. Studies on animals have shown that within the brain, BACE1 is enriched in synaptic vesicles [11]. Protease inhibition resulted consistently in higher levels of BACE1 being preserved in saliva. As with all tests, saliva sampling should follow a concise and practical protocol, allowing comparison within the same laboratory and patient setting. We established a protocol consisting of fasting saliva sampling before breakfast, with saliva allowed to pool in the anterior part of the mouth behind the front teeth with the mouth held open for 30 to 60 seconds. Saliva was removed with flexible plastic non sterile plastic pipettes and transferred to $1.5 \mathrm{ml}$ plastic sample vials and stored on ice immediately. Vials with the saliva sample then should be frozen within 1 hour. No anti-protease was added, as early saliva samples could not be pipetted accurately due to foaming.

All salivary samples tested revealed content of BACE 1 biomarker. Content of BACE1 varied considerably during daytime and with selfsampling by the patients. In a separate study, this variability could be lessened by our sampling protocol and by doing the sampling by a nurse or technician. Contrary to blood as biomarker, saliva reacts quite closely to changes in CNS. BACE1 is certainly implicated in the $\mathrm{AD}$ process and thus our results with elevated BACE1 saliva levels associated with $\mathrm{AD}$ (separate, not published to date) and with beginning cognitive impairment in our group of patients confirm the value of saliva as biomarker as well as the connection between elevated BACE1 levels in saliva with cognitive impairment and AD. This will have to be confirmed with a bigger number of patients, but is in itself 
logical and plausible. Other researchers [3] have found that BACE1 levels in blood platelets are lowered (sic!) in AD patients, which does just not seem logical.

Based on these test we have set a cut offvalue for BACE1 saliva content in $\mathrm{AD}$ patients, patientsat risk and normal patients (Table 5). These values should be considered laboratory specific and should be validated freshly when used in another setting. We also believe that we can predict AD or at least a higher risk to develop $\mathrm{AD}$ at a very early time, giving hope to prevent further accumulation of amyloid aggregates and even clear existing amyloid aggregates with stem cell therapy.

Table 5. Proposed Cut-off values for BACE1 saliva values.

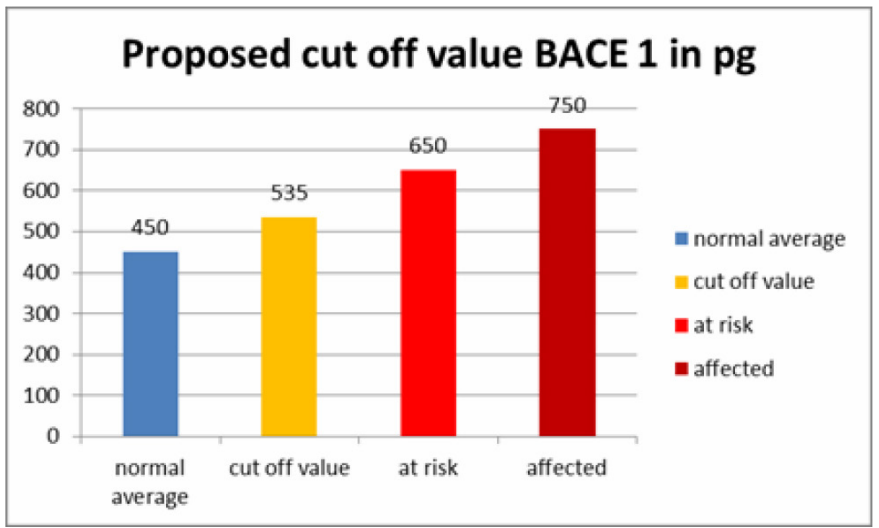

Two of our patients had stem cell secretome treatments before and after saliva sampling. The secretome is a patented, cell free product generated from the active ingredients which stem cells secrete as cell to cell communication or as a result of being stressed by various degrees of hypoxia or by preconditioning of the stem cell media before the production of the secretome. The secretome can thus be adapted to different diseases, as we have shown in other studies, i.e. beneficial for cartilage regeneration or with more content of neuroregenerative substances. One of the patients was in the group of at risk patients to develop $\mathrm{AD}$ and one patient had normal saliva values of BACE1. They both received intramuscular secretome 4 times at intervals of 2-4 weeks. Salivary BACE1 levels sank dramatically in both patients (Table 4). This confirms the treatment potential of $\mathrm{AD}$ with the secretome, although it is not clear which of the ingredients play a major role in this. As a total BACE1 knockout in rodents also lead to neurological dysfunction [8], a total block of BACE1 does not seem beneficial. However, the significant lowering of BACE1 over a long period of time would at least prevent new formation of amyloid beta aggregates and give to body a chance to clear aggregated proteins via the ubiquitin protease system. UCH-L1 in the secretome not only inhibits neuritic plaque formation, but also accelerates degradation of BACE1, regulates APP and is a major component of the ubiquitin protease system $[12,13]$.

It is still early days, and we are expanding our stem cell preventative treatments to the treatment of clinically manifest cognitive impairment and Alzheimer disease. In a better world, prevention of diseases, be it under the heading of anti-ageing or anti-morbidity, would be the preferred method of choice. It will be interesting to see, if the regenerative capacity of mesenchymal stem cells and their secretome will be sufficient to treat $\mathrm{AD}$ patients in advanced stages as well. The saliva biomarker BACE1 will give us a tool to monitor disease progression or regression, before clinical signs become evident.

\section{References}

1. McKhann GM, Knopman DS, Chertkow H, Hyman BT, Jack CR Jr, et al. (2011) The diagnosis of dementia due to Alzheimer's disease: recommendations from the National Institute on Aging-Alzheimer's Association workgroups on diagnostic guidelines for Alzheimer's disease. Alzheimers Dement 7: 263-269. [Crossref]

2. Zhou Y, Tan C, Wen D, Sun H, Han W, et al. (2016) The Biomarkers for Identifying Preclinical Alzheimer's Disease via Structural and Functional Magnetic. Front Aging Neurosci 8: 92. [Crossref]

3. Koyama A, Okereke OI, Yang T, Blacker D, Selkoe DJ, et al. (2012) Plasma Amyloid-beta as a Predictor of Dementia and Cognitive Decline: A Systematic Review and Meta-analysis. Arch Neurol 69: 824-31. [Crossref]

4. Selkoe DJ (2011) Alzheimer's Disease. Cold Spring Harb Perspect Biol 3: a004457. [Crossref]

5. Bayer TA, Wirths O (2010) Intracellular accumulation of amyloidBeta - a predictor for synaptic dysfunction and neuron loss in Alzheimer's disease. Front Aging Neurosci 2: 8. [Crossref]

6. Roberds SL, Anderson J, Basi G, Bienkowski MJ, Branstetter DG, et al. (2001) BACE knockout mice are healthy despite lacking the primary beta-secretase activity in brain: implications for Alzheimer's disease therapeutics. Hum Mol Genet 10: 1317-1324. [Crossref]

7. Sun X, He G, Song W (2006) BACE2, as a novel APP thetasecretase, is not responsible for the pathogenesis of Alzheimer's disease in Down syndrome. FASEB J 20: 1369-1376. [Crossref]

8. Munro KM, Nash A, Pigoni M, Lichtenthaler SF, Gunnersen JM (2016) Functions of the Alzheimer's Disease Protease BACE1 at the Synapse in the Central Nervous System. J Mol Neurosci 60: 305-315. [Crossref]

9. Devi L, Ohnu M (2015) A combination Alzheimer's therapy targeting BACE1 and Neprilysin in 5XFAD transgenic mice. Mol Brain 8: 19. [Crossref]

10. Zhang M, Deng Y, Luo Y, Zhang S, Zou H, et al. (2012) Control of BACE1 degradation and APP processing by ubiquitin carboxyl-terminal hydrolase L1. $J$ Neurochem 120: 1129-1138. [Crossref]

11. Lundgren JL, Ahmed S, Schedin-Weiss S, Gouras GK, Winblad B et al. (2015) ADAM10 and BACE1 are localized to synaptic vesicles. J Neurochem 135: 606615. [Crossref]

12. Tramutola A, Di Domenico F, Barone E, Perluigi M, Butterfield DA (2016) It is all about Ubiquitin: Role of altered Ubiquitin-Proteasome System and UCHL1 in Alzheimer Disease. Oxid Med Cell Longev. [Crossref]

13. Gong B, Radulovic M, Figueiredo-Pereira ME, Cardozo C (2016) The UbiquitinProteasome System: Potential Therapeutic Targets for Alzheimer Disease and Spinal Cord Injury. Front Mol Neurosci 9: 4. [Crossref]

\section{Citation:}

SJ Kellner (2018) Diagnosis and monitoring of Alzheimer Disease with saliva biomarker BACE1 and Preliminary study on prevention of AD with mesenchymal stem cells and their secretome. Ageing Sci Ment Health Stud Volume 2(2): 1-4 